OPEN ACCESS

Edited by:

Jue Zhang,

Peking University, China

Reviewed by:

Inga Griskova-Bulanova,

Vilnius University, Lithuania

Antonino Naro,

Centro Neurolesi Bonino Pulejo

(IRCCS), Italy

Karin Diserens,

Centre Hospitalier Universitaire

Vaudois (CHUV), Switzerland

*Correspondence:

Nai Ding

ding_nai@zju.edu.cn

Benyan Luo

luobenyan@zju.edu.cn

tThese authors have contributed equally to this work and share first

authorship

Specialty section: This article was submitted to

Neural Technology, a section of the journa

Frontiers in Neuroscience

Received: 27 December 2020 Accepted: 19 March 2021

Published: 28 April 2021

Citation:

Xu C, Zou J, He F, Wen X, Li J,

Gao J, Ding N and Luo B (2021)

Neural Tracking of Sound Rhythms Correlates With Diagnosis, Severity, and Prognosis of Disorders

of Consciousness.

Front. Neurosci. 15:646543. doi: 10.3389/fnins.2021.646543

\section{Neural Tracking of Sound Rhythms Correlates With Diagnosis, Severity, and Prognosis of Disorders of Consciousness}

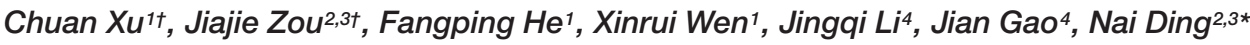 \\ and Benyan Luo ${ }^{1 *}$
}

${ }^{1}$ Department of Neurology, First Affiliated Hospital, School of Medicine, Zhejiang University, Hangzhou, China, ${ }^{2}$ Key Laboratory for Biomedical Engineering of Ministry of Education, College of Biomedical Engineering and Instrument Sciences, Zhejiang University, Hangzhou, China, ${ }^{3}$ Research Center for Advanced Artificial Intelligence Theory, Zhejiang Lab, Hangzhou, China, ${ }^{4}$ Department of Rehabilitation, Hangzhou Mingzhou Brain Rehabilitation Hospital, Hangzhou, China

Effective diagnosis and prognosis of patients with disorders of consciousness (DOC) provides a basis for family counseling, decision-making, and the design of rehabilitation programs. However, effective and objective bedside evaluation is a challenging problem. In this study, we explored electroencephalography (EEG) response tracking sound rhythms as potential neural markers for DOC evaluation. We analyzed the responses to natural speech and tones modulated at 2 and $41 \mathrm{~Hz}$. At the population level, patients with positive outcomes (DOC-P) showed higher cortical synchronization to modulated tones at $41 \mathrm{~Hz}$ compared with patients with negative outcomes (DOC$\mathrm{N})$. At the individual level, phase coherence to modulated tones at $41 \mathrm{~Hz}$ was significantly correlated with Coma Recovery Scale-Revised (CRS-R) and Glasgow Outcome Scale-Extended (GOS-E) scores. Furthermore, SVM classifiers, trained using phase coherences in higher frequency bands or combination of the low frequency aSSR and speech tracking responses, performed very well in diagnosis and prognosis of DOC. These findings show that EEG response to auditory rhythms is a potential tool for diagnosis, severity, and prognosis of DOC.

Keywords: disorders of consciousness, neural synchronization, auditory steady state response, EEG, machine learning

\section{INTRODUCTION}

Prolonged disorders of consciousness (DOC) is a group of neurological syndromes caused by severe brain damage, in which impairment of consciousness lasts more than 28 days from onset (Giacino et al., 2018). Prolonged DOC patients include patients in vegetative state (VS)/unresponsive wakefulness syndrome (UWS) and patients in minimally conscious state (MCS). VS/UWS is a condition of wakefulness without awareness (Laureys et al., 2010). These patients may have their eyes open but exhibit reflex behaviors only; therefore, they are considered unaware of themselves and their surroundings (Naccache, 2018). On the contrary, MCS patients show unequivocal signs of non-reflex cortically mediated behaviors in response to environmental stimuli, 
which occur inconsistently but are reproducible (Giacino et al., 2002). The Coma Recovery Scale-Revised (CRS-R) is a behavioral assessment method recommended to determine the level of consciousness in DOC (Estraneo et al., 2015; Iazeva et al., 2018; Kondziella et al., 2020). However, determining consciousness in unresponsive patients by behavioral assessment is challenging because patients must be awake during evaluation. In addition, patients must possess the voluntary drive to mobilize motor function. This trait must be preserved to a degree that is readily measurable (Kondziella et al., 2020). Furthermore, origin of most clinical signs and behaviors in DOC has not been fully explored and their correlation with patient consciousness is not fully known, making the method unreliable (Schnakers et al., 2009; Cortese et al., 2015; van Erp et al., 2015). These limitations and unavailability of adequate diagnostic tools result in approximately $40 \%$ misdiagnosis cases in DOC (Stender et al., 2014).

Several neuroimaging technologies have been proposed for assessing brain activity, such as MRI (Bardin et al., 2011; Monti et al., 2015; Edlow et al., 2017), PET (Stender et al., 2014, 2016), and fNIRS (Kurz et al., 2018; Abdalmalak et al., 2020) to circumvent the limitations of traditional diagnostic methods. Previous studies recommend integration of standardized clinical evaluation, electroencephalography (EEG)based techniques, and functional neuroimaging to achieve multimodal evaluation of DOC (Kondziella et al., 2020). However, functional neuroimaging is not widely available and it is not clinically feasible for use in large numbers of patients. EEG is an attractive option as it is portable, cost-effective, and relatively feasible to deploy at the patient's bedside (Chennu et al., 2017). Recent cross-sectional studies report that the brain networks, based on bedside high density electroencephalography (hdEEG) at rest, can be used to predict brain metabolic changes, diagnosis, and prognosis of DOC (Chennu et al., 2014, 2017; Wu et al., 2020). A previous longitudinal study reports that functional brain networks based on bedside hdEEG are an important prognosis predictor for DOC (Bareham et al., 2020). Moreover, the auditory Steady State Response (aSSR) is a promising tool for diagnosis and prognosis of DOC (Gorska and Binder, 2019).

When listening to sound with temporal modulations, neural activity can synchronize to the modulations. In human EEG and MEG recordings, such neural synchronization is most salient in two frequency bands. One frequency occurs around $40 \mathrm{~Hz}$ (Ross et al., 2000) and the other frequency occurs below $10 \mathrm{~Hz}$ (Wang et al., 2012). Neural synchronization to temporal modulations around $40 \mathrm{~Hz}$ is observed when listening to tones amplitude or frequency modulated at $40 \mathrm{~Hz}$ (Luo et al., 2006; Ding and Simon, 2009; Millman et al., 2010), and such responses are usually referred as the $40 \mathrm{~Hz}$ aSSR (Galambos et al., 1981). Neural synchronization to slow modulations below $10 \mathrm{~Hz}$ is observed when listening to tones modulated at a slow rate or when listening to natural speech (Ding and Simon, 2012b; Peelle et al., 2013; Doelling et al., 2014; Harding et al., 2019). Previous studies report that the phase-locking index of $40 \mathrm{~Hz}$ aSSR is an indicator of the level of central nervous system dysfunction in DOC (Binder et al., 2017, 2020). Further, previous studies report that neural response is observed in the sideband which reflects interaction between AM and FM response (Luo et al., 2006). In this study, we analyzed the response at $40 \mathrm{~Hz}$ and in sideband at $41 \pm 2 \mathrm{~Hz}$. Similar to high-frequency stimulus-synchronized responses to modulated tones, it is also found that neural synchronization at low rates can be used as an objective estimate of the level of neural dysfunction in DOC patients (Gorska and Binder, 2019).

Previous studies report that neural synchronization to speech is mainly observed in delta and theta bands for language conditions (Luo et al., 2006; Ding and Simon, 2012b, 2014). Notably, theta-band neural response and delta-band neural response plays different functions. For instance, theta-band response encodes syllabic-level acoustic features critical for speech recognition, whereas delta-band response is related to the perceived non-speech-specific acoustic rhythm (Ding and Simon, 2014). Studies have also explored speech tracking responses in DOC and show that speech tracking response is progressively delayed across healthy individuals, MCS patients, and UWS patients (Braiman et al., 2018). In this study, we explored the combination of multiple passive EEG paradigms, including neural synchronization to temporal modulations and speech tracking responses, to improve diagnosis and prognosis of DOC.

Herein, we recorded the $40 \mathrm{~Hz}$ aSSR, the low-frequency stimulus-synchronized responses to modulated tones, and speech tracking responses in the same groups of participants, aimed at elaborating the clinical utility of the three responses in DOC. In addition, we analyzed the potential of the three responses in diagnosis and prognosis of DOC.

\section{MATERIALS AND METHODS}

\section{Subjects and Neurobehavioral Assessments}

A total of 47 subjects were included in this study. The subjects included 16 healthy individuals, 15 MCS patients, and 16 UWS patients. Five CRS-R assessments were performed in DOC patients 10 days before EEG recording. Diagnosis of the patients was based on the highest score of five CRSR assessments (Wannez et al., 2017; Kondziella et al., 2020). Detailed information on patients, including sex, age, etiology, diagnosis, CRS-R results, time after the injury, and GOS-E results are presented in Supplementary Table 1. Two evaluations using CRS-R (before the experiment, 6 months later) and one evaluation using GOS-E (6 months after the experiment) were carried out and were also presented in Supplementary Table 1. The inclusion criteria for this study were as follows: (i) diagnosis with UWS, MCS based on the highest score of 5 assessments carried out for a period of 10 days by DOC experts using CRSR (Kondziella et al., 2020); (ii) patients who had stayed for more than 1 month after brain injury; (iii) patients with no history of hearing impairment before brain injury; (iv) patients not under centrally acting drugs, neuromuscular function blockers, or sedation within $24 \mathrm{~h}$ prior to the study, patients should not have visible skull bone defects (CT); (v) patients with no history of neurodegenerative diseases such as Alzheimer's disease and Parkinson's disease before brain injury. Follow-up of behavioral measurements (CRS-R and GOS-E) of patients was carried out for more than 6 months after EEG assessments. An informed 
consent was acquired from their legal surrogates for all patients, whereas all health individuals signed written informed consent. The study was approved by the Ethical Committee of the First Affiliated Hospital of Zhejiang University and Hangzhou Mingzhou Brain Rehabilitation Hospital.

\section{Procedures}

All participants listened to speech and modulated tones while EEG responses were recorded. Natural speech included two chapters from the novel The Supernova Era by Cixin Liu (Chapter 16: Fun country and Chapter 18: Sweet dream period). The story was narrated in Mandarin by a female speaker. Narration of the two chapters took $34 \mathrm{~min}$ and $25 \mathrm{~min}$, respectively. The two chapters were presented sequentially (first Chapter 16, then Chapter 18). Modulated tones were sinusoidally frequency modulated at $2 \mathrm{~Hz}$ and sinusoidally amplitude modulated at $41 \mathrm{~Hz}$. Speech and modulated tones were both presented binaurally through headphones. The experiment was performed in two separate days. All participants had their eyes open at the beginning of the experiment on each day. The spoken narrative was presented on the first day of the experiment. The modulated tones were presented after the spoken narrative. On the second day of the experiment, the same spoken narrative presented on day 1 was presented. The 59-min speech stimulus was repeated and therefore the total speech stimulus took approximately $2 \mathrm{~h}$, which was longer compared with the stimulus duration in most studies. A long stimulus was used to effectively estimate the response phase. Healthy individuals and EMCS patients were asked to keep still during the experiment. No other tasks were allowed during the experiment; therefore, the participants listened passively. Data collection on the second day was terminated by the participant or the legal surrogate for three MCS patients and one healthy individual, therefore, for these participants we only analyzed the response to modulated tones.

\section{EEG Data Collection}

Electroencephalography signals were recorded using a 64electrodes BrainCap (Brain Products DmbH, Munich, Germany) in the international 10-20 system while listening to the auditory materials, and 1 of the 64 electrodes was placed under the right eye to record electrooculogram (EOG). EEG signals were referenced online to $\mathrm{FCz}$, but were referenced offline to a common average reference. All signals were sampled at $1 \mathrm{kHz}$ with a $50 \mathrm{~Hz}$ zero-phase Butterworth notch filter applied online.

\section{EEG Data Analysis}

Electroencephalography recordings were low-pass filtered below $50 \mathrm{~Hz}$ with a zero-phase anti-aliasing FIR filter (implemented using a $200 \mathrm{~ms}$ Kaiser window) and down-sampled to $100 \mathrm{~Hz}$. EOG artifacts were regressed out based on the least-squares method. Speech tracking EEG responses were expected to be similar across the two presentations of the same story; therefore, EEG recordings were averaged across recording days. Cerebroacoustic phase coherence spectrum was calculated to characterize neural synchronization to auditory stimuli (modulated tones or speech) (Peelle et al., 2013). EEG response and acoustic envelope were segmented into non-overlapping $2 \mathrm{~s}$ time bins, and all segments were converted into the frequency domain using Discrete Fourier Transform (DFT). Cerebro-acoustic phase coherence was a function of frequency, and it quantified synchronization between sound envelope and neural response in each frequency bin. It was formulated as

$$
C_{f}=\left(\sum_{t=1}^{T} \cos \left(\alpha_{f t}-\beta_{f t}\right)\right)^{2}+\left(\sum_{t=1}^{T} \sin \left(\alpha_{f t}-\beta_{f t}\right)\right)^{2}
$$

where $\alpha_{f t}$ and $\beta_{f t}$ independently denote the response phase and stimulus phase in frequency bin $f$ and time bin $t$. $C_{f}$ is the phase coherence in frequency bin $f$. Higher phase coherence indicated that the response phase was more precisely synchronized to the stimulus phase. Based on the Rayleigh test for phase coherence (Fisher, 1993), if the $C_{f}$ is larger than three, the phase distribution is significantly different from a uniform distribution $(P<0.05)$.

For the modulated tone, the period of the stimulus was $0.5 \mathrm{~s}$. Therefore, in each $2 \mathrm{~s}$ time bin, the stimulus was identical and therefore $\beta_{f t}$ was a constant. In this case, the calculation of $C_{f}$ reduced to

$$
C_{f}=\left(\sum_{t=1}^{T} \cos \left(\alpha_{f t}\right)\right)^{2}+\left(\sum_{t=1}^{T} \sin \left(\alpha_{f t}\right)\right)^{2}
$$

which is similar to the inter-trial phase coherence (Luo and Poeppel, 2007). Phase coherence was strongest in central-frontal electrodes; therefore, 14 centro-frontal electrodes, i.e., Fz, F1, F2, F3, F4, FC1, FC2, FC3, FC4, Cz, C1, C2, C3, and C4, were used to compare the phase coherence value across populations and to perform correlation analysis and SVM classification.

\section{Response Topography}

Phase coherence was always positive and measured how strongly the response phase was synchronized to the stimulus phase. However, when analyzing the response topography, it was useful to show whether the responses in different EEG electrodes were in phase or out of phase. Therefore, a signed phase coherence, a simplified version of the complex-valued topography, was considered in the response topography analysis (Simon and Wang, 2005). Specifically, channel Fz was chosen as a reference since it showed strongest phase coherence in most conditions. For each electrode, if the phase difference between this electrode and electrode $\mathrm{Fz}$ was larger than $90^{\circ}$, the phase coherence was negated. On the contrary, if the phase difference between this electrode and electrode $\mathrm{Fz}$ was less than $90^{\circ}$, the phase coherence was kept positive. The signed phase coherence can illustrate the phase relationship between electrodes on top of showing the phase coherence.

\section{Statistical and Classification Analysis}

To evaluate whether response phase coherence at one frequency was significantly higher than chance, we first estimated the chance-level distribution of phase coherence.

In the responses to modulated tones, since responses were expected at integer frequencies, the responses at non-integer frequencies, i.e., $0.5,1.5, \ldots, 49.5 \mathrm{~Hz}$, were considered as chancelevel phase coherence. Furthermore, the test was applied to the 
group response; therefore, if the group included $\mathrm{N}$ participants, we estimated the chance-level distribution by randomly selecting $\mathrm{N}$ participants from all 47 participants and averaged the responses over the $\mathrm{N}$ participants. This sampling procedure was repeated 1,000 times. The phase coherence from the 1,000 times at the 50 non-integer frequencies were pooled. If the response coherence at one frequency was lower than the Ns of the 50,000 chance-level coherence values (one-sided comparison), its significance value was calculated as Ns/50,000. This procedure was used instead of the Rayleigh test to evaluate the significance of phase coherence since the phase coherence was averaged over channels and participants, whereas the Rayleigh test applies to data in a single channel from a single participant.

Bias-corrected and accelerated bootstrap was used for the unpaired comparisons between groups in Figure $\mathbf{1}$ (Efron and Tibshirani, 1994). Data from each group were resampled 5,000 times with replacement. This comparison was also one-sided. If in $\mathrm{N}$ out of 5,000 times, the mean in one group was greater (or smaller) than the other group, the significance level was $(N+1) / 5,001$. Correlational analyses were performed using twotailed bivariate Pearson correlation coefficient. In this study, correlation analysis was performed between CRS-R score, GOS-E score, and the phase coherence. The single outlier was removed from correlation analysis to ensure reliability of the results.

Two-class support vector machine (SVM) classifiers were trained by phase coherence to modulated tones and natural speech to determine whether the neural synchronization to fast and slow auditory rhythms are potential application in diagnosis and prognosis of DOC. SVM is a classic machine-learning model used for classification using both small and large sample sizes (Way et al., 2010). For structural dependence models SVM, the special principle and algorithm is to find a hyper-plane in high dimensional space with feature vectors from the samples. Therefore, SVM was used in this study as an effective prediction model for classification of variables using the limited sample (Zhang et al., 2018). Radial basis function (RBF) was used as the SVM kernel function, which is the most common kernel function used to map data into a space (Gromski et al., 2014). Samples were divided randomly into training and test sets to determine the generalization of the model. Approximately 70\% of UWS and MCS patients were randomly selected as training sets to build the model. A grid search method within a five-fold crossvalidation procedure was used to optimize the regularization parameter of the SVM model (Cost and $\gamma$ ). The remaining 30\% of patients were used as a test set to validate the function. Phase coherence at 41 and $41 \pm 2 \mathrm{~Hz}$, phase coherence at 2 and $4 \mathrm{~Hz}$, phase coherence for the speech tracking response in delta and theta bands, combination of the phase coherence at 2 and $4 \mathrm{~Hz}$, phase coherence to natural speech in delta, theta bands were used to train the SVM classifiers. Phase coherence at 41 and $41 \pm 2 \mathrm{~Hz}$, phase coherence at 2 and $4 \mathrm{~Hz}$ from 31 patients including of $15 \mathrm{MCS}$ patients and 16 UWS patients were used in DOC classification analysis. The EEG data of the speech tracking response of three MCS patients were incomplete; therefore, phase coherence for the speech tracking response in delta and theta bands, the combination of phase coherence at 2 and $4 \mathrm{~Hz}$, phase coherence for speech tracking response in delta, theta bands for
28 patients, including $12 \mathrm{MCS}$ patients and 16 UWS patients, were used in the DOC classification analysis.

Follow-up of behavioral measurements (CRS-R and GOSE) of patients was carried out for more than 6 months after EEG assessments for prediction of patient outcome. Each patient was initially labeled as showing a positive or negative outcome. Clinical diagnoses of patients were subcategorized into four different subclasses based on proposed ascending levels of consciousness, namely UWS, MCS-, MCS+, and EMCS. In this study, positive outcome was defined as any advances in transition of clinical categorization during follow-up, whereas negative outcome was defined as stasis or retrogress in transition. Phase coherence at 41 and $41 \pm 2 \mathrm{~Hz}$, phase coherence at 2 and $4 \mathrm{~Hz}$ of 31 patients including 17 patients with positive outcomes and 14 patients with negative outcomes were used in prognosis prediction analysis. Three MCS patients were excluded due to incomplete EEG data of the speech tracking response. Therefore, phase coherence for the speech tracking response in delta and theta bands, combination of phase coherence at 2 and $4 \mathrm{~Hz}$, phase coherence for the speech tracking response in delta, theta bands of 28 patients, including 15 patients with positive outcomes and 13 patients with negative outcomes were used in prognosis prediction analysis. Diagnosis and prediction analysis in this study were conducted using the python program.

\section{RESULTS}

\section{Neural Synchronization to Auditory Rhythms in DOC With Good and Poor Prognosis}

To explore neural synchronization to auditory rhythms in DOC with good and poor prognosis, patients with positive prognosis (DOC-P) were considered as a separate group, patients with poor prognosis (DOC-N) were grouped as another separate group, then phase coherence between neural responses and acoustic envelope in the group of healthy controls (HC), DOC$\mathrm{P}$, and DOC-N was calculated. The results in Figure 1 were averaged across all electrodes. As shown in Figure 1, phase coherences to modulated tones at 41 and $41 \pm 2 \mathrm{~Hz}$ were significant in HC, DOC-P, and DOC-N, and there seemed to be no difference for phase coherences to modulated tones at 41 and $41 \pm 2 \mathrm{~Hz}$ between $\mathrm{HC}$ and DOC-P. However, the difference of speech tracking response in delta and theta bands between the group of healthy controls (HC) and DOC-P were observed. Analysis of topography of the neural synchronization to auditory rhythms showed a centro-frontal distribution for all 3 groups of participants (Figure 2). Therefore, 14 centro-frontal channels were selected for further analyses. Phase coherence averaged across centro-frontal electrodes were compared across populations as shown in Figure 3. Phase coherence to modulated tones at 41 and $41 \pm 2 \mathrm{~Hz}$ for $\mathrm{HC}$ group was not significantly different compared with that of the DOC-P group. However, phase coherences at 41 and at $41 \pm 2 \mathrm{~Hz}$ in the HC and DOC-P groups were significantly higher compared with that in the DOC$\mathrm{N}$ group $(P<0.01$, bootstrap, FDR corrected) (Figures 3A,D). 

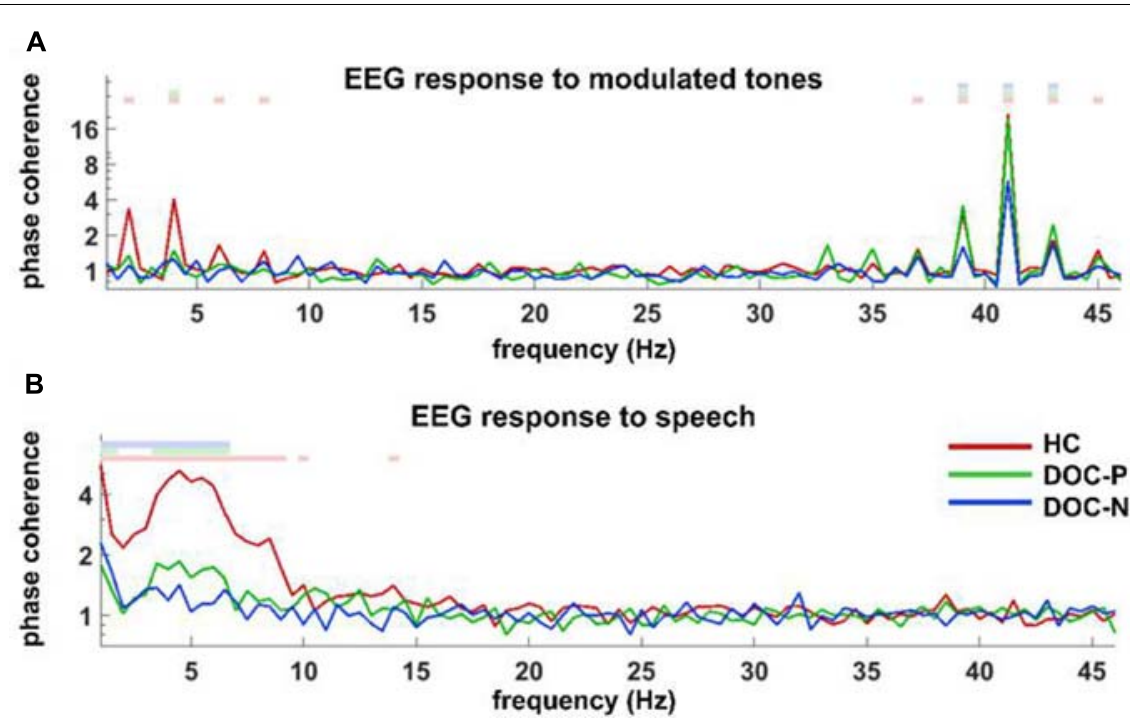

FIGURE 1 | Phase coherence spectrum for responses to modulated tones $\mathbf{( A )}$ and speech $\mathbf{( B )}$ in the group of healthy controls (HC), patients with positive prognosis (DOC-P), and patients with negative prognosis (DOC-N). (A) Coherences to modulated tones at 41 and $41 \pm 2 \mathrm{~Hz}$ were significant in $\mathrm{HC}$, DOC-P, DOC-N, and there seemed to be no difference between $\mathrm{HC}$ and DOC-P. (B) Speech tracking response in delta and theta frequency bands for the group of healthy controls ( $\mathrm{HC}$ ) was significantly different from that of DOC-P group. The colored squares on top denote the frequency bins in which phase coherence is significantly higher compared with chance $(P<0.01$, bootstrap, FDR corrected).

Phase coherences for DOC-P at 2 and $4 \mathrm{~Hz}$ were not significantly different compared with those for DOC-N group $(P>0.05$, bootstrap, FDR corrected) (Figures 3B,E). For speech tracking response, phase coherences to natural speech in theta and delta bands for HC group were significantly different compared with those for DOC-P group $(P<0.01$, bootstrap, FDR corrected) (Figures 3C,F).

\section{Correlations Between Neural Synchronization and the Clinical Behaviors}

To evaluate whether neural synchronization to temporal modulations and natural speech had values to determine the diagnosis, severity, and prognosis of DOC, we set out to assess the correlations between the neural synchronization and clinical assessments of neurological function in individual patients. A single outlier was removed before performing correlation analysis to ensure the reliability of the results. Phase coherence at $41 \mathrm{~Hz}$ was independently correlated with CRS-R and GOSE $(r=0.59, P=0.011$, FDR corrected; $r=0.5, P=0.027$, FDR corrected) (Figure 4). Furthermore, phase coherence at $41 \pm 2 \mathrm{~Hz}$ was correlated with CRS-R ( $r=0.55, P=0.015$, FDR corrected) (Figure 4). Analysis showed that correlations between the phase coherence at 2 and $4 \mathrm{~Hz}, \mathrm{CRS}-\mathrm{R}$, and GOS-E were not significant (Figure 5). In addition, correlation between phase coherence to natural speech in lower frequency bands and CRS-R and correlation between phase coherence to natural speech in lower frequency bands and GOS-E were not significant (Figure 6). Details on correlation coefficient analysis between CRS-R total score, GOS-E total score, and cerebro-acoustic phase coherence are shown in Table $\mathbf{1 .}$

\section{Classification and Prognosis Prediction Based on Neural Synchronization in DOC}

To further analyze the role of neural synchronization in diagnosis and prognosis of DOC, two-class SVM classifiers were trained using the multiple EEG measurements. SVM classifiers were trained separately using phase coherences at 41 and $41 \pm 2 \mathrm{~Hz}$, phase coherences at 2 and $4 \mathrm{~Hz}$, phase coherences for speech tracking response in delta and theta bands, combination of phase coherences at 2 and $4 \mathrm{~Hz}$, and phase coherences for the speech tracking response in delta, theta bands.

SVM classifiers were trained by phase coherences to modulated tones at 41 and $41 \pm 2 \mathrm{~Hz}$ (Figures 7A,B). The confusion matrix showed that the classifier trained by phase coherence at 41 and $41 \pm 2 \mathrm{~Hz}$ showed $86.67 \%$ sensitivity and $87.5 \%$ specificity in discriminating MCS and UWS $\left(\chi^{2}=17.052\right.$, $P=0.001$, accuracy $=70 \%$, AUC $=87.08 \%$, Figure 7A). In prognosis prediction of DOC, the SVM classifier trained by phase coherences at 41 and $41 \pm 2 \mathrm{~Hz}$ performed very well $\left(\chi^{2}=14.072, P=0.001\right.$, accuracy $=90 \%$, AUC $\left.=83.4 \%\right)$ with high sensitivity (88.24\%) and specificity $(78.57 \%)$ in positive prognosis prediction (Figure 7B). However, SVM classifiers trained by phase coherence at 2 and $4 \mathrm{~Hz}$ did not perform well in classification and prognosis prediction of DOC (Figures 7C,D).

A two-class SVM classifier was trained by phase coherence to natural speech in delta and theta bands to analyze the role of speech tracking response in classification and prognosis prediction of DOC. The inputs to the classifier were delta and theta speech tracking response. The confusion matrix in Figure $\mathbf{8 A}$, generated by the SVM classifier trained by the phase coherence to the natural speech in delta and theta bands, 

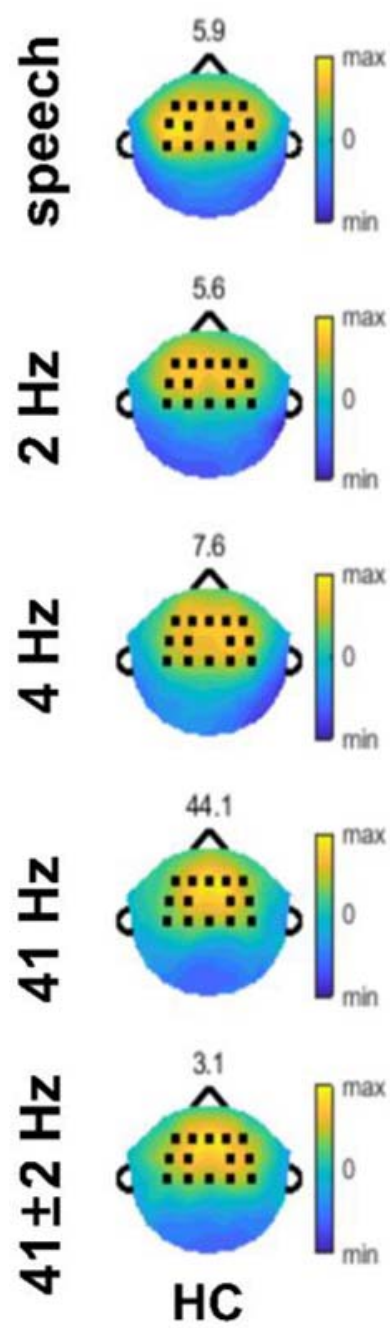
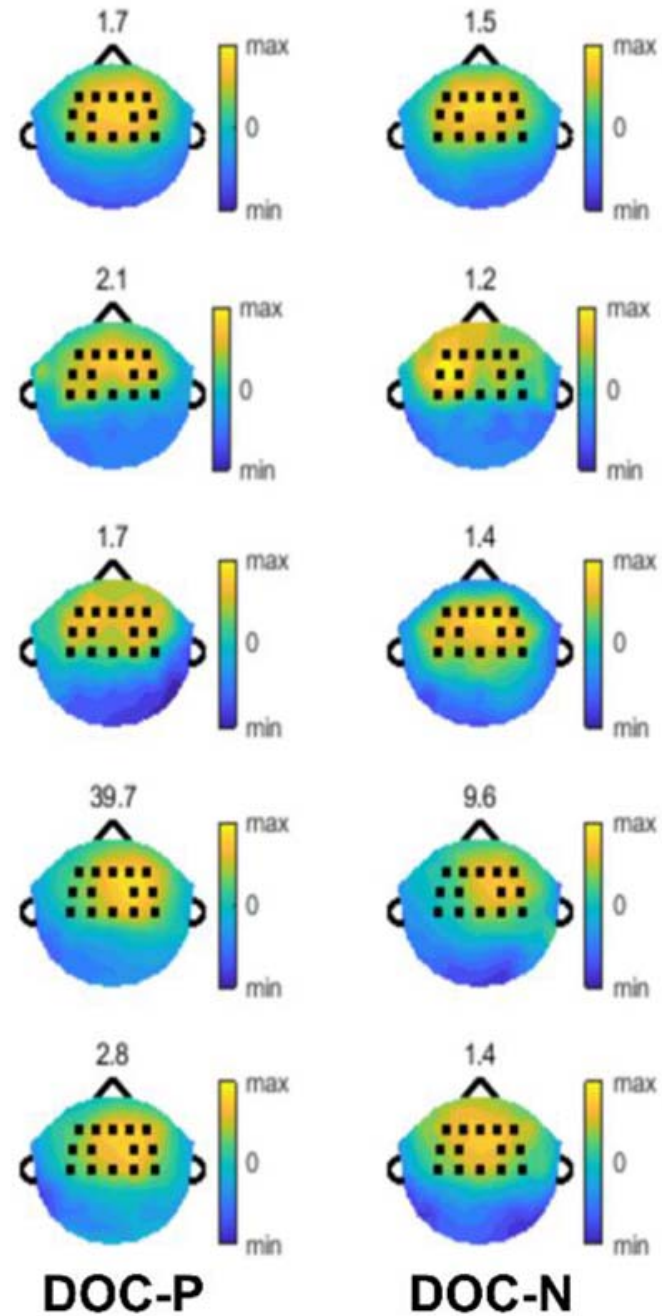

FIGURE 2 | Topography of phase coherence. Phase coherence was separately normalized for each population by dividing by the 95th percentile of phase coherence across electrodes, to accurately illustrate the spatial distribution, and the values of the 95th percentile are shown on top of each plot.

showed that the classifier had $50 \%$ sensitivity and $100 \%$ specificity in discriminating MCS and UWS $\left(\chi^{2}=10.182, P=0.001\right.$, accuracy $=70 \%$, AUC $=75 \%$, Figure 8A). However, this classifier did not perform well in prognosis prediction $\left(\chi^{2}=3.387\right.$, $P=0.084$, accuracy $=66.67 \%$, AUC $=66.15 \%$, Figure 8B).

Further, the classifier was trained using a combination of phase coherences at 2 and $4 \mathrm{~Hz}$, and phase coherences for the speech tracking response in delta, theta bands. Analysis of the classifier showed $75 \%$ sensitivity and $87.5 \%$ specificity in discriminating MCS and VS $\left(\chi^{2}=11.23\right.$, $P=0.001$, accuracy $=88.89 \%$, AUC $=81.25 \%$, Figure 8C). In addition, the SVM classifier predicted positive prognosis of the individual patients with high sensitivity $(86.67 \%)$ and specificity $(76.92 \%)\left(\chi^{2}=11.449, P=0.002\right.$, accuracy $=77.78 \%$, AUC $=81.79 \%$, Figure 8D). The performance of the SVM classifiers in classification and prognosis prediction of DOC was significantly higher using combined training of phase coherences at 2 and $4 \mathrm{~Hz}$, and phase coherences for speech tracking response in delta, theta bands, compared with training using separate parameters.

\section{DISCUSSION}

Physical and cognitive impairments experienced by individuals with DOC vary greatly, and it is difficult to distinguish behaviors that are indicative of conscious awareness from those that are random and non-purposeful, making diagnosis and prognosis challenging. Previous studies report that multimodal evaluations have high sensitivity in detection of consciousness (Giacino et al., 2018). In this study, the role of multiple passive EEG paradigms in the diagnosis and prognosis of DOC was explored. The neural synchronization to auditory rhythms in DOC patients with good and poor prognosis was analyzed. Phase coherences at 41 and $41 \pm 2 \mathrm{~Hz}$ in DOC-N were significantly lower compared with those of $\mathrm{HC}$ and DOC-P. However, phase coherences 

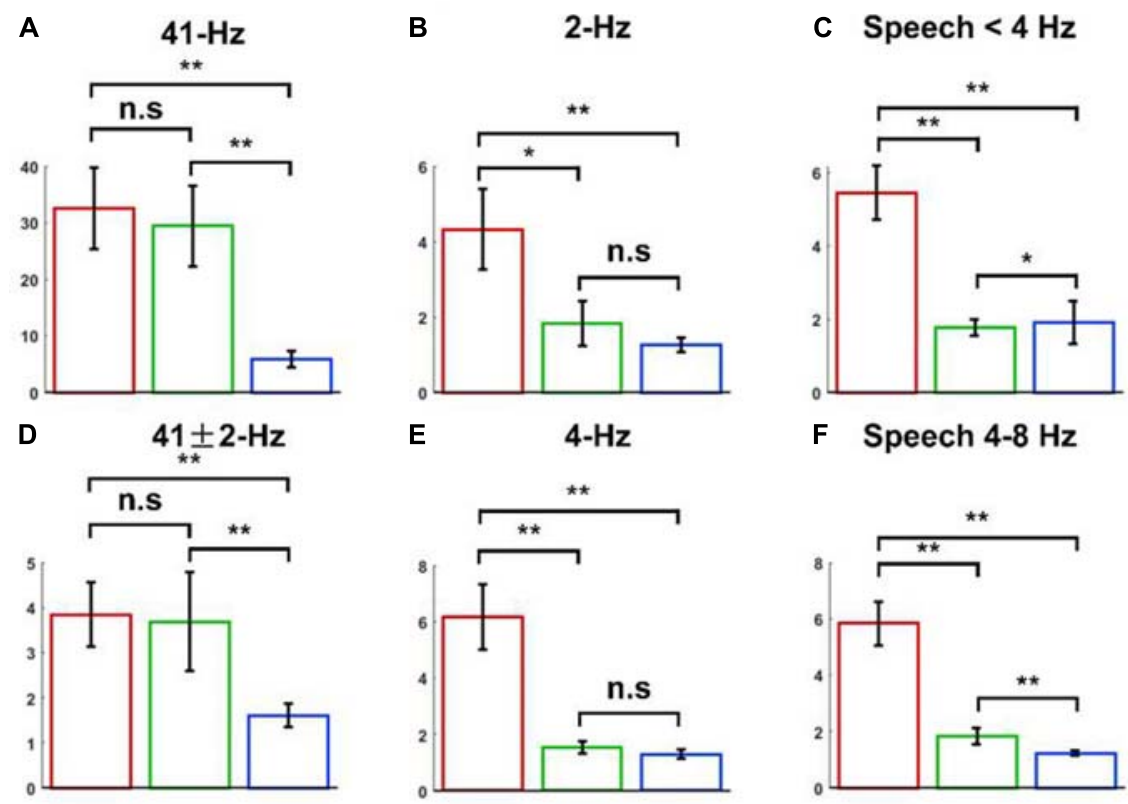

E

4- $\mathrm{Hz}$
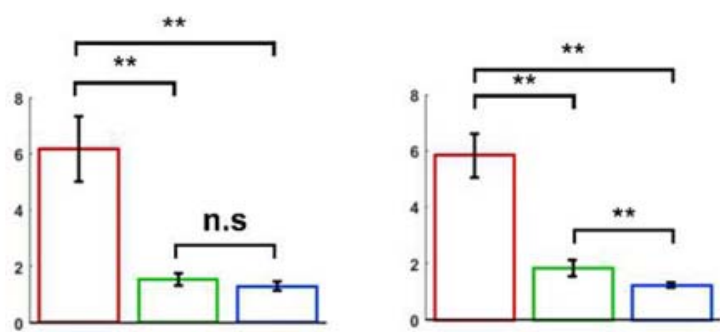

HC

\section{DOC-P}

DOC-N

FIGURE 3 | Mean and SEM of the phase coherence between neural responses and acoustic envelope in the healthy controls (HC), patients with positive prognosis (DOC-P), and patients with negative prognosis (DOC-N). (A,D) There were no statistically significant differences in the 41 and $41 \pm 2 \mathrm{~Hz}$ phase coherences between for $\mathrm{HC}$ and DOC-P. However, the 41 and $41 \pm 2 \mathrm{~Hz}$ phase coherences were significantly higher in HC and DOC-P groups compared with those for DOC-N group. (B,E) The 2 and $4 \mathrm{~Hz}$ phase coherences in $\mathrm{HC}$ were significantly higher compared with those for DOC-P. (C,F) Phase coherences to natural speech in theta and delta bands were significantly different between $\mathrm{HC}$ and DOC-P groups ( ${ }^{\star} P<0.05$, ${ }^{\star \star} P<0.01$; n.s., not significant).
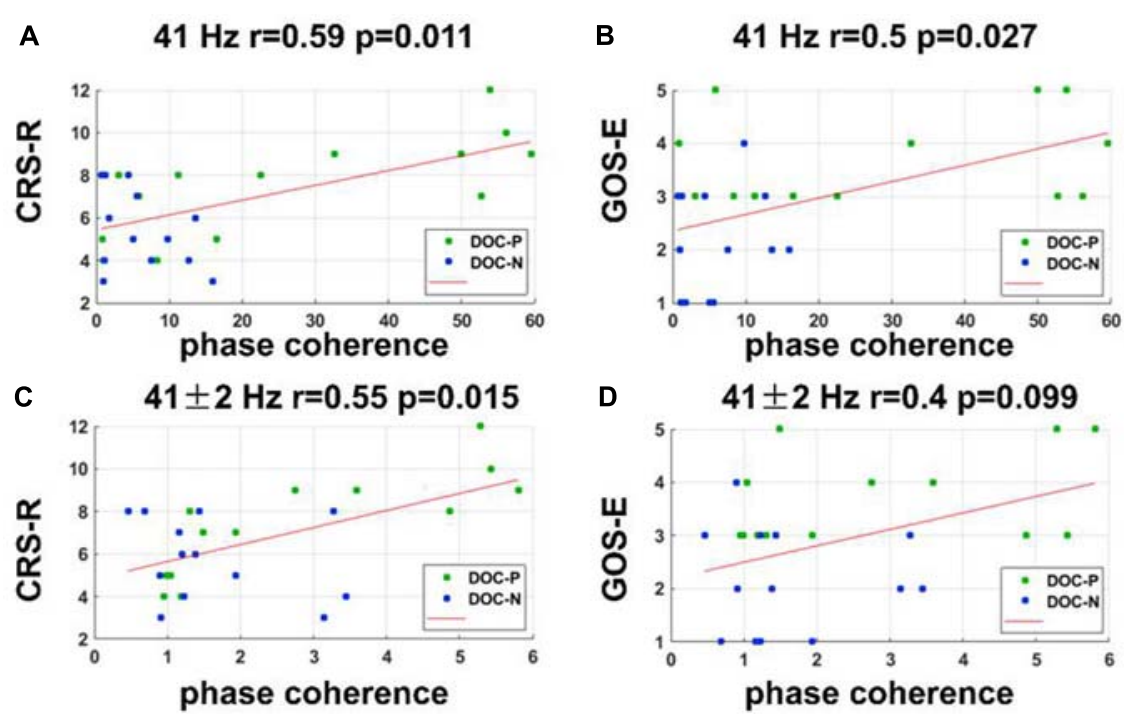

FIGURE 4 | Correlations between neural synchronization at $41,41 \pm 2 \mathrm{~Hz}$, and clinical evaluations. (A,B) Correlations between the phase coherence at $41 \mathrm{~Hz}$ and CRS-R, and GOS-E scores. Phase coherence at $41 \mathrm{~Hz}$ was independently correlated with CRS-R and GOS-E scores $(r=0.59, P=0.011$, FDR corrected; $r=0.5$, $P=0.027$, FDR corrected). (C,D) Correlations between the phase coherence at $41 \pm 2 \mathrm{~Hz}$ and CRS-R, and GOS-E scores. Phase coherence at $41 \pm 2 \mathrm{~Hz}$ was correlated with CRS-R score $(r=0.55, P=0.015$, FDR corrected).

at 41 and $41 \pm 2 \mathrm{~Hz}$ of $\mathrm{HC}$ were not significantly different from those of DOC-P group. This finding implies that DOC patients with normal aSSR in higher frequency bands may retain specific aspects of cerebral function related to prognosis. Further, correlation analysis showed that neural synchronization to modulated tones at $41 \mathrm{~Hz}$ in DOC was correlated with GOS-E, 

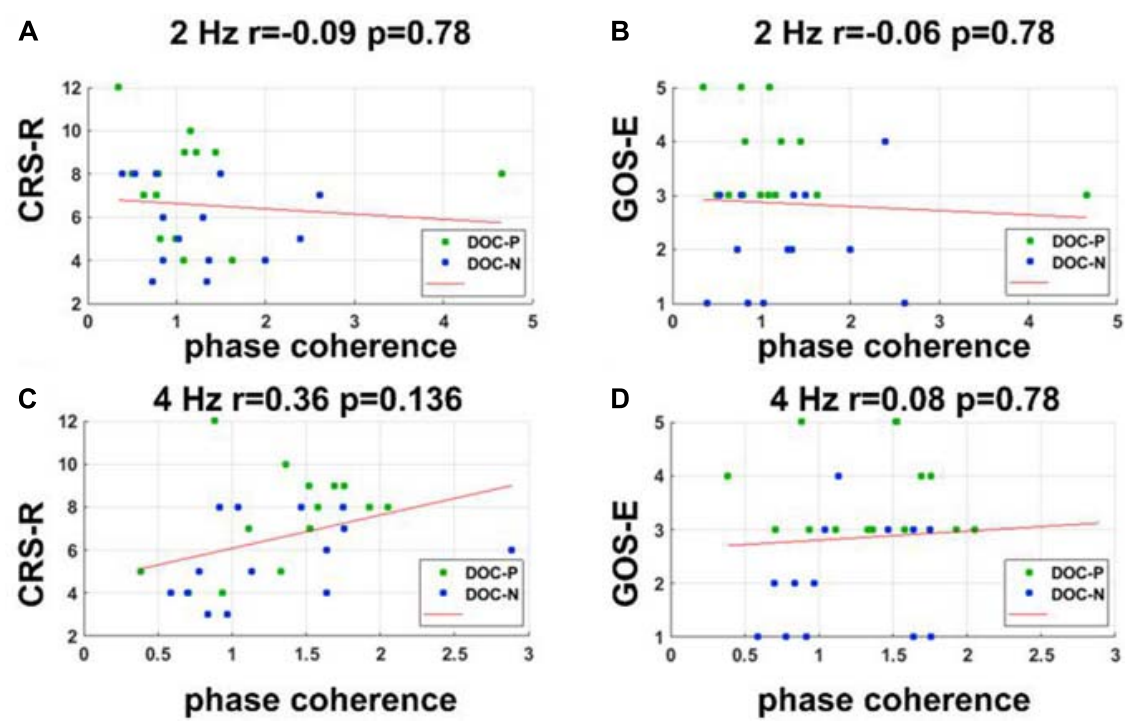

FIGURE 5 | Correlations between neural synchronization at 2 and $4 \mathrm{~Hz}$, and clinical evaluations. (A-D) Correlations between phase coherence at 2 , 4 Hz, and CRS-R, GOS-E scores. Correlations between the phase coherence at 2, $4 \mathrm{~Hz}$, and CRS-R, GOS-E were not statistically significant.
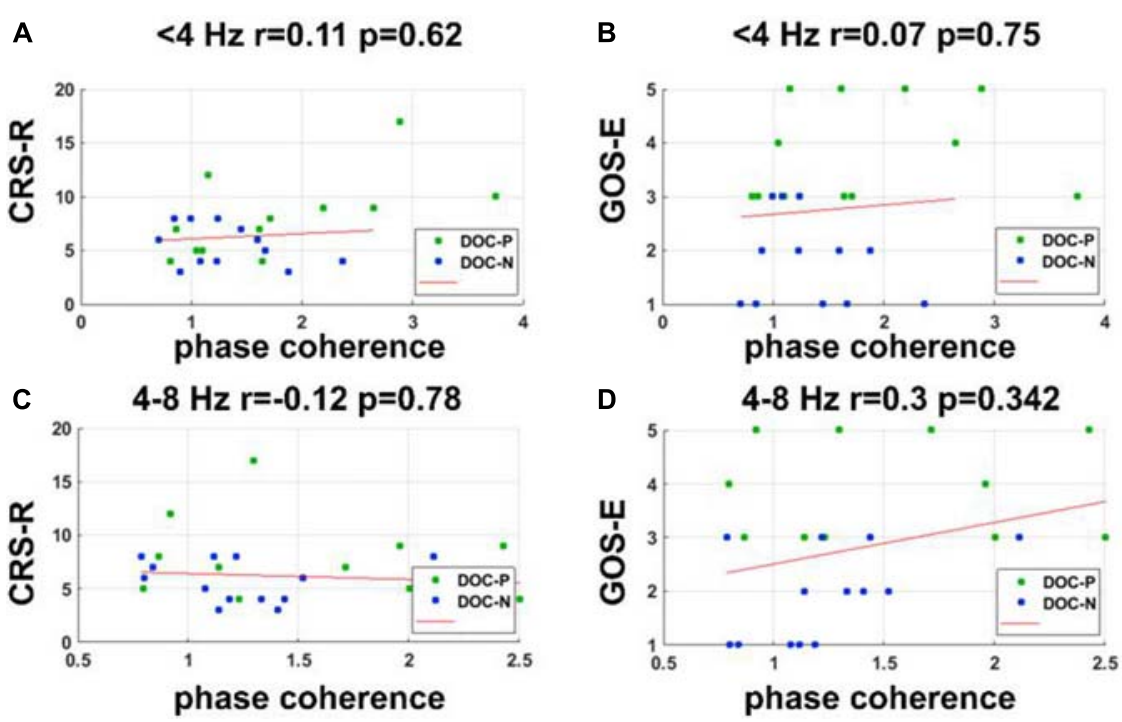

FIGURE 6 | Correlations between the phase coherence for the speech tracking response in delta and theta bands and clinical evaluations. (A-D) Correlations between the phase coherence for the speech tracking response in delta, theta bands, and CRS-R, GOS-E score showed no statistical significance.

TABLE 1 | Correlations between CRS-R total score, GOS-E total score, and cerebro-acoustic phase coherence (FDR corrected). Significant $P$-values (<0.05) are shown in bold.

\begin{tabular}{|c|c|c|c|c|c|c|c|c|c|c|c|c|}
\hline & \multicolumn{2}{|c|}{$41-\mathrm{Hz}$} & \multicolumn{2}{|c|}{$41 \pm 2-H z$} & \multicolumn{2}{|c|}{$2 \mathrm{~Hz}$} & \multicolumn{2}{|c|}{$4 \mathrm{~Hz}$} & \multicolumn{2}{|c|}{ Speech <4 Hz } & \multicolumn{2}{|c|}{ Speech 4-8 Hz } \\
\hline & $r$ & $p$ & $r$ & $p$ & $r$ & $p$ & $r$ & $p$ & $r$ & $p$ & $r$ & $p$ \\
\hline Total CRS-R score & 0.59 & 0.011 & 0.55 & 0.015 & -0.09 & 0.78 & 0.36 & 0.136 & 0.11 & 0.62 & -0.12 & 0.78 \\
\hline Total GOS-E score & 0.5 & 0.027 & 0.4 & 0.099 & -0.06 & 0.78 & 0.08 & 0.78 & 0.07 & 0.75 & 0.3 & 0.342 \\
\hline
\end{tabular}

the scoring scale used for prognosis of DOC. Moreover, SVM classifier was trained using phase coherence at 41 and $41 \pm 2 \mathrm{~Hz}$. Analysis of the results showed that the classifier predicted positive prognosis of individual patients with high sensitivity and specificity. For speech tracking response, SVM classifier was trained using delta and theta speech tracking response and 

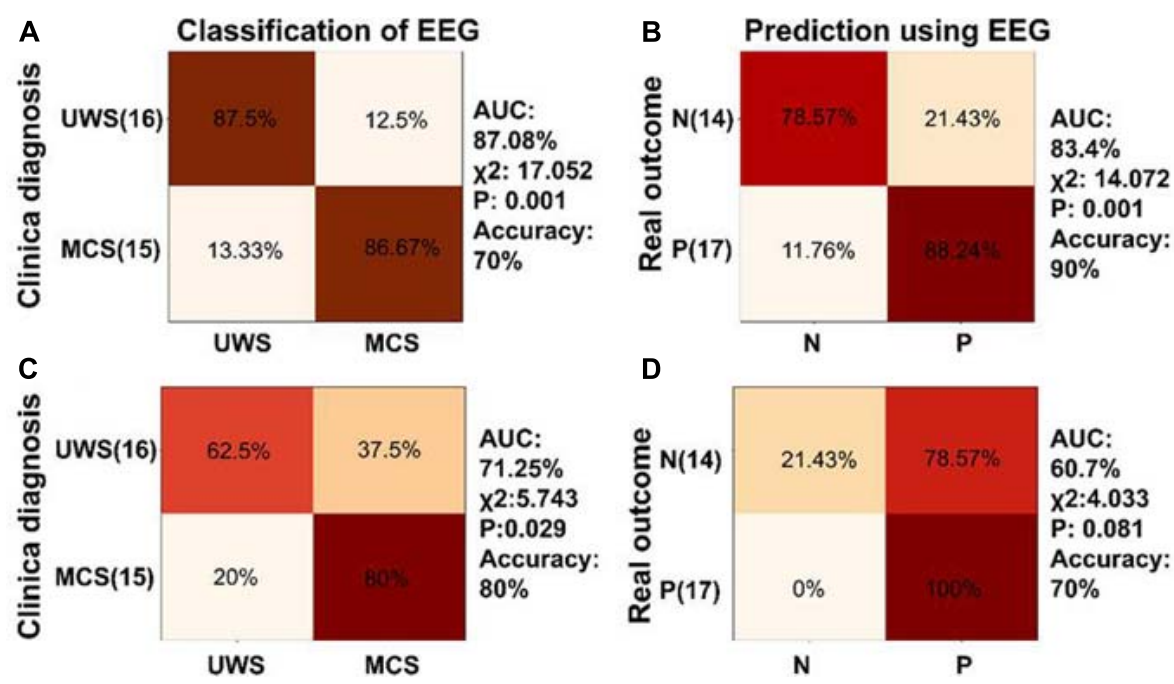

FIGURE 7 | Classification and prognosis prediction in DOC using neural synchronization to temporal modulations at low and high frequencies. (A) Diagnosis classification using phase coherence at 41 and $41 \pm 2 \mathrm{~Hz}$ performed well (AUC $=87.08 \%$, accuracy $=70 \%, \chi^{2}=17.052, P=0.001$ ). Confusion matrix generated by SVM showed $86.67 \%$ sensitivity and $87.5 \%$ specificity for MCS diagnosis. (B) The SVM classifier trained using phase coherence at 41 and $41 \pm 2 \mathrm{~Hz}$ showed good performance in prognosis prediction of DOC ( $A \cup C=83.4 \%$, accuracy $=90 \%, \chi 2=14.072, P=0.001$ ) and was able to predict positive prognosis of individual patients with high sensitivity $(88.24 \%)$ and specificity $(78.57 \%)$. (C,D) The SVM classifier showed poor performance in classification and prognosis prediction of DOC trained using phase coherence at 2 and $4 \mathrm{~Hz}$.
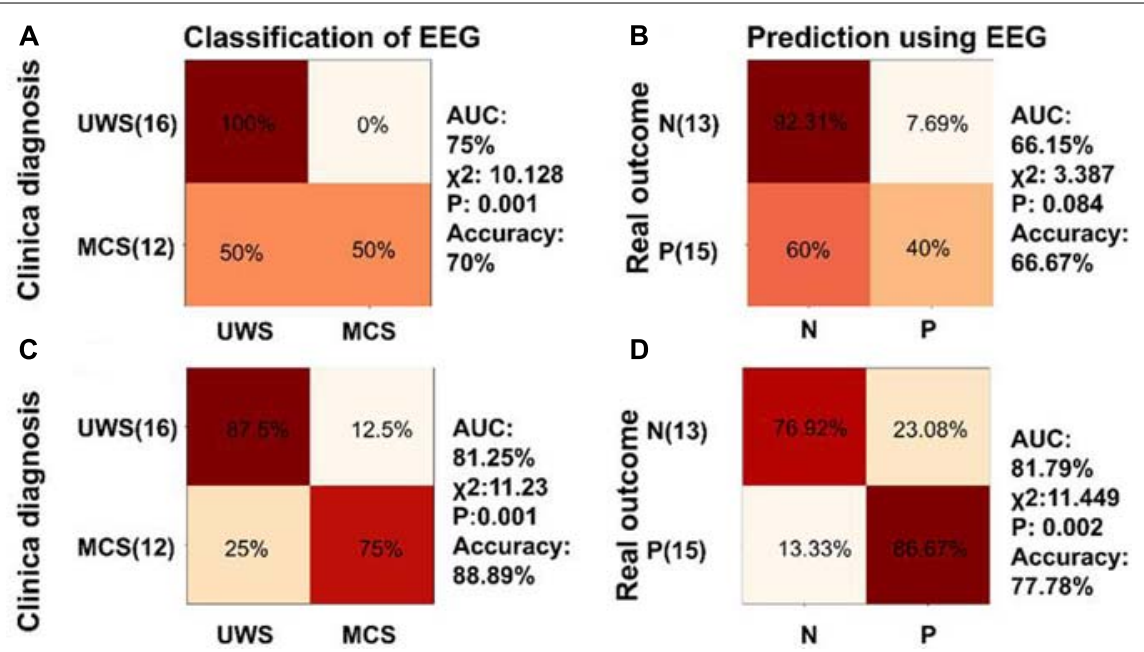

FIGURE 8 | Classification and diagnosis prediction using speech tracking response and combination of low frequency aSSR and speech tracking responses. (A,B) Confusion matrix generated by the SVM classifier trained by the phase coherence to the natural speech in delta and theta bands for classification and prognosis prediction of DOC. (C,D) Confusion matrix generated by the SVM classifier trained by the combination of the phase coherence to modulated tones at 2, $4 \mathrm{~Hz}$, and the phase coherence to natural speech in delta, theta bands for classification and prognosis prediction of DOC. The SVM classifiers showed better performance in classification and prognosis prediction of DOC compared with SVM classifiers separately trained with phase coherence at 2 and $4 \mathrm{~Hz}$ or the phase coherence to natural speech in delta and theta bands.

analysis showed that the classifier accurately classified MCS and UWS. Notably, SVM classifiers trained using a combination of phase coherences at 2 and $4 \mathrm{~Hz}$, and phase coherences for the speech tracking response in delta, theta bands showed better performance in classification and prognosis prediction of DOC, compared with training using the separate parameters.

The $40 \mathrm{~Hz}$ aSSR and low-frequency auditory responses below $10 \mathrm{~Hz}$ reflect neural processing on different time scales and are likely to be generated from different neural sources. The $40 \mathrm{~Hz}$ aSSR belongs to the gamma band and is generated in primary auditory cortex and auditory midbrain and thalamus (Ross et al., 2005; Steinmann and Gutschalk, 2011). Previous studies report that progressive deafferentation in neurons within the central thalamus is in proportion to the severity of structural brain injuries, and central thalamus plays a crucial role in the maintenance of consciousness (Maxwell et al., 2006; 
Giacino et al., 2014). Therefore, DOC patients can be classified by detecting $40 \mathrm{~Hz}$ aSSR that reflects thalamic function. Previous studies also report that $40 \mathrm{~Hz}$ aSSR is positively correlated with CRS-R total score and with the scores of the Auditory and Visual subscales in DOC (Binder et al., 2017, 2020). In this study, analysis showed that $40 \mathrm{~Hz}$ aSSR was correlated with CRS-R total score. Moreover, the $40 \mathrm{~Hz}$ aSSR was positively correlated with the GOS-E score. Furthermore, the SVM classifier trained using the aSSR at 41 and $41 \pm 2 \mathrm{~Hz}$, accurately classified MCS and UWS and predicted the good and poor prognosis in DOC. These findings imply that aSSR at high frequency has broad application prospects in diagnosis and prognosis of DOC. Lowfrequency stimulus-synchronized responses and speech tracking responses in delta and theta bands are generated from more broad cortical areas in the temporal and frontal lobes (Ding and Simon, 2012a; Zion Golumbic et al., 2013). In this study, low-frequency stimulus-synchronized responses to modulated tones showed no value in diagnosis and prognosis of DOC.

Recent studies show that speech tracking responses are progressively delayed along with the awareness decline in healthy individuals, MCS patients, and UWS patients, and may help to identify cognitive motor dissociation (CMD) in DOC (Braiman et al., 2018). In this study, SVM classifier was trained using delta and theta speech tracking response. Analysis showed that the classifier accurately distinguished MCS from UWS. A recent study using isochronously presented speech reports that EEG-derived neural signals, including speechtracking responses and temporal dynamics of global brain states, are associated with behavioral diagnosis of consciousness and are accurate in prediction of future outcomes in individual patients (Gui et al., 2020). In this study, analysis showed that speech tracking response is a potential indicator for distinguishing MCS from UWS.

In this study, a passive paradigm was used to explore whether neural tracking of sound rhythms is useful in determining residual consciousness in DOC. Compared with passive paradigms, cognitive demands of active paradigms are higher. Although successful demonstration of covert commandfollowing is a widely accepted clinical marker of awareness and useful for prognosis, its sensitivity is compromised by precluding many patients with cognitive deficits from demonstrating the extent of their abilities (Guger et al., 2003; Monti et al., 2010). Furthermore, passive paradigm is more convenient and practical in clinical applications compared with active paradigm. A positive response to stimulation is covert cortical processing in passive paradigms. Notably, ability of covert cortical processing to reflect residual consciousness process depends on the types of passive paradigm. However, a positive response in active paradigms may reflect CMD.

A number of active paradigm studies report that patients with severe brain injury may not reveal any signs of consciousness at the bedside, but some of them are able to willfully modulate their brain activity on command, even occasionally answering yes/no questions by performing mental imagery tasks (Monti et al., 2010). Approximately $15 \%$ of behaviorally VS/UWS patients are able to follow commands by modifying their brain activity during an EEG- and/or fMRI-based active consciousness paradigm, implying that they have covert cognitive abilities (Kondziella et al., 2016). CMD defines patients who demonstrate sharp dissociation of an inability or extremely limited ability to move with preservation of higher-level cognition in the form of reliable command-following, as detected with functional MRI, EEG, or other non-invasive measures (Schiff, 2015). Emerging evidence indicates that CMD patients represent a distinct subgroup of patients with DOC, whose brain networks and clinical features might fundamentally differ from those of other subgroups (Pincherle et al., 2019; Edlow et al., 2020; Johr et al., 2020). Pan et al. (2020) report that patients with CMD have a better outcome compared with other patients (Pan et al., 2020). A previous study reported that cortical response to the natural speech envelope can help identify CMD (Braiman et al., 2018). CMD patients should be differentiated from DOC patients for determination of the application of various paradigms in diagnosis and prognosis in DOC more objectively and effectively in the future.

Currently, multimodal evaluation of DOC, integrated with EEG-based techniques and functional neuroimaging, is recommended as it is highly accurate (Kondziella et al., 2020). Approaches for detecting consciousness by means of positron emission tomography (PET), fMRI, and EEG have been developed in the past two decades to supplement clinical evaluation of DOC (Gosseries et al., 2014; Kondziella et al., 2016; Marino et al., 2016). The default mode network (DMN) is absent in brain death; however, it is partially preserved in VS, probably reflecting residual structural connectivity (Soddu et al., 2012). Several studies report the prognostic value of bloodoxygen-level-dependent (BOLD) signals elicited by a subject's own name in traumatic VS (Wang et al., 2015). These studies report that functional neuroimaging has important implications for clinical diagnosis and prognosis for patients with disorders of consciousness. However, functional neuroimaging is not widely available and may not be clinically feasible in large numbers of patients. Combination of multiple passive EEG paradigms is more valuable for diagnosis and prognosis of DOC. The results in this study were consistent with the hypothesis: the performance of the SVM classifier trained by combination of phase coherence at 2 and $4 \mathrm{~Hz}$, phase coherence for the speech tracking response in delta, theta bands performed better compared with the classifier separately trained using phase coherence at 2 and $4 \mathrm{~Hz}$, or phase coherence for the speech tracking response in delta, theta bands. Multimodal evaluation based on a combination of multiple passive EEG paradigms has important implications and is more feasible for clinical diagnosis and prognosis of patients with disorders of consciousness.

However, this study had some limitations. In order to ensure the credibility of the research results, the inclusion criteria of subjects were relatively strict and prevalence rate of DOC was relatively low. These factors result in insufficient sample size in this study. To further verify these results, multi-center studies for neural synchronization to fast and slow auditory rhythms should be conducted in the future with larger sample sizes. Neural tracking of sound rhythms showed the potential in diagnosis and prognosis in DOC; however, the normal value range was not determined. In addition, heterogeneity of DOC etiology may affect the accuracy of the results. Further studies should be 
performed using patients with DOC caused by single etiology, such as traumatic brain injury.

In summary, analysis of phase coherences to natural speech and modulated tones at 2 and $41 \mathrm{~Hz}$ shows that EEG responses to auditory rhythms is a potential tool for predicting diagnosis, severity, and prognosis of DOC.

\section{DATA AVAILABILITY STATEMENT}

The original contributions presented in the study are included in the article/Supplementary Material, further inquiries can be directed to the corresponding author/s.

\section{ETHICS STATEMENT}

The studies involving human participants were reviewed and approved by the Ethical Committee of the First Affiliated Hospital of Zhejiang University and Hangzhou Mingzhou Brain Rehabilitation Hospital. The patients/participants provided their written informed consent to participate in this study. Written informed consent was obtained from the individual(s) for the publication of any potentially identifiable images or data included in this article.

\section{AUTHOR CONTRIBUTIONS}

CX: investigation, data curation, and writing - review and editing. JZ: methodology, software, formal analysis, and writing -

\section{REFERENCES}

Abdalmalak, A., Milej, D., Yip, L. C. M., Khan, A. R., Diop, M., Owen, A. M., et al. (2020). Assessing time-resolved fNIRS for brain-computer interface applications of mental communication. Front Neurosci 14:105. doi: 10.3389/ fnins.2020.00105

Bardin, J. C., Fins, J. J., Katz, D. I., Hersh, J., Heier, L. A., Tabelow, K., et al. (2011). Dissociations between behavioural and functional magnetic resonance imaging-based evaluations of cognitive function after brain injury. Brain 134, 769-782. doi: 10.1093/brain/awr005

Bareham, C. A., Roberts, N., Allanson, J., Hutchinson, P. J. A., Pickard, J. D., Menon, D. K., et al. (2020). Bedside EEG predicts longitudinal behavioural changes in disorders of consciousness. Neuroimage Clin. 28:102372. doi: 10. 1016/j.nicl.2020.102372

Binder, M., Gorska, U., and Griskova-Bulanova, I. (2017). 40Hz auditory steadystate responses in patients with disorders of consciousness: Correlation between phase-locking index and Coma Recovery Scale-Revised score. Clin. Neurophysiol. 128, 799-806. doi: 10.1016/j.clinph.2017.02.012

Binder, M., Gorska, U., Pipinis, E., Voicikas, A., and Griskova-Bulanova, I. (2020). Auditory steady-state response to chirp-modulated tones: a pilot study in patients with disorders of consciousness. Neuroimage Clin. 27:102261. doi: 10.1016/j.nicl.2020.102261

Braiman, C., Fridman, E. A., Conte, M. M., Voss, H. U., Reichenbach, C. S., Reichenbach, T., et al. (2018). Cortical response to the natural speech envelope correlates with neuroimaging evidence of cognition in severe brain injury. Curr. Biol. 28, 3833-3839.e3.

Chennu, S., Annen, J., Wannez, S., Thibaut, A., Chatelle, C., Cassol, H., et al. (2017). Brain networks predict metabolism, diagnosis and prognosis at the bedside in disorders of consciousness. Brain 140, 2120-2132. doi: 10.1093/brain/awx163 review and editing. $\mathrm{FH}$ : visualization and writing - review and editing. XW: visualization, resources, and writing review and editing. JL and JG: investigation, resources, and data curation. ND: conceptualization, methodology, software, formal analysis, resources, writing - review and editing, and supervision. BL: conceptualization, resources, supervision, project administration, and writing - review and editing. All authors contributed to the article and approved the submitted version.

\section{FUNDING}

This work was supported by the National Natural Science Foundation of China 81870817, Guangzhou Key R\&D Program of China 202007030005, and National Key R\&D Program of China 2018YFA0701400.

\section{ACKNOWLEDGMENTS}

The authors thank the patients, their families, and volunteers for participation in this study.

\section{SUPPLEMENTARY MATERIAL}

The Supplementary Material for this article can be found online at: https://www.frontiersin.org/articles/10.3389/fnins. 2021.646543/full\#supplementary-material

Chennu, S., Finoia, P., Kamau, E., Allanson, J., Williams, G. B., Monti, M. M., et al. (2014). Spectral signatures of reorganised brain networks in disorders of consciousness. PLoS Comput. Biol. 10:e1003887. doi: 10.1371/journal.pcbi. 1003887

Cortese, M. D., Riganello, F., Arcuri, F., Pugliese, M. E., Lucca, L. F., Dolce, G., et al. (2015). Coma recovery scale-r: variability in the disorder of consciousness. BMC Neurol 15:186. doi: 10.1186/s12883-015-0455-5

Ding, N., and Simon, J. Z. (2009). Neural representations of complex temporal modulations in the human auditory cortex. J. Neurophysiol. 102, 2731-2743. doi: 10.1152/jn.00523.2009

Ding, N., and Simon, J. Z. (2012a). Emergence of neural encoding of auditory objects while listening to competing speakers. Proc. Natl. Acad. Sci. U.S.A. 109, 11854-11859. doi: 10.1073/pnas.1205381109

Ding, N., and Simon, J. Z. (2012b). Neural coding of continuous speech in auditory cortex during monaural and dichotic listening. J. Neurophysiol. 107, 78-89. doi: $10.1152 /$ jn.00297.2011

Ding, N., and Simon, J. Z. (2014). Cortical entrainment to continuous speech: functional roles and interpretations. Front. Hum. Neurosci. 8:311. doi: 10.3389/ fnhum.2014.00311

Doelling, K. B., Arnal, L. H., Ghitza, O., and Poeppel, D. (2014). Acoustic landmarks drive delta-theta oscillations to enable speech comprehension by facilitating perceptual parsing. Neuroimage 85(Pt 2), 761-768. doi: 10.1016/j. neuroimage.2013.06.035

Edlow, B. L., Chatelle, C., Spencer, C. A., Chu, C. J., Bodien, Y. G., O’Connor, K. L., et al. (2017). Early detection of consciousness in patients with acute severe traumatic brain injury. Brain 140, 2399-2414. doi: 10.1093/brain/awx176

Edlow, B. L., Claassen, J., Schiff, N. D., and Greer, D. M. (2020). Recovery from disorders of consciousness: mechanisms, prognosis and emerging therapies. Nat. Rev. Neurol. 17, 135-156. doi: 10.1038/s41582-020-00428-x 
Efron, B., and Tibshirani, R. J. (1994). An Introduction to the Bootstrap. London: Chapman and Hall.

Estraneo, A., Moretta, P., De Tanti, A., Gatta, G., Giacino, J. T., Trojano, L., et al. (2015). An Italian multicentre validation study of the coma recovery scale-revised. Eur. J. Phys. Rehabil. Med. 51, 627-634.

Fisher, N. I. (1993). Statistical Analysis of Circular Data: Descriptive methods. Cambridge: Cambridge University Press.

Galambos, R., Makeig, S., and Talmachoff, P. J. (1981). A 40-Hz auditory potential recorded from the human scalp. Proc. Natl. Acad. Sci. U.S.A. 78, 2643-2647. doi: $10.1073 /$ pnas.78.4.2643

Giacino, J. T., Ashwal, S., Childs, N., Cranford, R., Jennett, B., Katz, D. I., et al. (2002). The minimally conscious state: definition and diagnostic criteria. Neurology 58, 349-353. doi: 10.1212/wnl.58.3.349

Giacino, J. T., Fins, J. J., Laureys, S., and Schiff, N. D. (2014). Disorders of consciousness after acquired brain injury: the state of the science. Nat. Rev. Neurol. 10, 99-114. doi: 10.1038/nrneurol.2013.279

Giacino, J. T., Katz, D. I., Schiff, N. D., Whyte, J., Ashman, E. J., Ashwal, S., et al. (2018). Practice guideline update recommendations summary: disorders of consciousness: report of the guideline development, dissemination, and implementation subcommittee of the American Academy of Neurology; the American Congress of Rehabilitation Medicine; and the National Institute on Disability, Independent Living, and Rehabilitation Research. Neurology 91, $450-460$.

Gorska, U., and Binder, M. (2019). Low- and medium-rate auditory steady-state responses in patients with prolonged disorders of consciousness correlate with Coma Recovery Scale - revised score. Int. J. Psychophysiol. 144, 56-62. doi: 10.1016/j.ijpsycho.2019.08.001

Gosseries, O., Di, H., Laureys, S., and Boly, M. (2014). Measuring consciousness in severely damaged brains. Annu. Rev. Neurosci. 37, 457-478. doi: 10.1146/ annurev-neuro-062012-170339

Gromski, P. S., Xu, Y., Correa, E., Ellis, D. I., Turner, M. L., and Goodacre, R. (2014). A comparative investigation of modern feature selection and classification approaches for the analysis of mass spectrometry data. Anal. Chim. Acta 829, 1-8. doi: 10.1016/j.aca.2014.03.039

Guger, C., Edlinger, G., Harkam, W., Niedermayer, I., and Pfurtscheller, G. (2003). How many people are able to operate an EEG-based brain-computer interface (BCI)? IEEE Trans. Neural Syst. Rehabil. Eng. 11, 145-147. doi: 10.1109/tnsre. 2003.814481

Gui, P., Jiang, Y., Zang, D., Qi, Z., Tan, J., Tanigawa, H., et al. (2020). Assessing the depth of language processing in patients with disorders of consciousness. Nat. Neurosci. 23, 761-770. doi: 10.1038/s41593-020-0639-1

Harding, E. E., Sammler, D., Henry, M. J., Large, E. W., and Kotz, S. A. (2019). Cortical tracking of rhythm in music and speech. Neuroimage 185, 96-101. doi: 10.1016/j.neuroimage.2018.10.037

Iazeva, E. G., Legostaeva, L. A., Zimin, A. A., Sergeev, D. V., Domashenko, M. A., Samorukov, V. Y., et al. (2018). A Russian validation study of the Coma Recovery Scale-Revised (CRS-R). Brain Inj. doi: 10.1080/02699052.2018. 1539248 [Epub ahead of print].

Johr, J., Halimi, F., Pasquier, J., Pincherle, A., Schiff, N., and Diserens, K. (2020). Recovery in cognitive motor dissociation after severe brain injury: a cohort study. PLoS One 15:e0228474. doi: 10.1371/journal.pone.0228474

Kondziella, D., Bender, A., Diserens, K., van Erp, W., Estraneo, A., Formisano, R., et al. (2020). European Academy of Neurology guideline on the diagnosis of coma and other disorders of consciousness. Eur. J. Neurol. 27, 741-756. doi: 10.1111/ene.14151

Kondziella, D., Friberg, C. K., Frokjaer, V. G., Fabricius, M., and Møller, K. (2016). Preserved consciousness in vegetative and minimal conscious states: systematic review and meta-analysis. J. Neurol. Neurosurg. Psychiatry 87, 485-492. doi: 10.1136/jnnp-2015-310958

Kurz, E. M., Wood, G., Kober, S. E., Schippinger, W., Pichler, G., MullerPutz, G., et al. (2018). Towards using fNIRS recordings of mental arithmetic for the detection of residual cognitive activity in patients with disorders of consciousness (DOC). Brain Cogn. 125, 78-87. doi: 10.1016/j.bandc.2018.06. 002

Laureys, S., Celesia, G. G., Cohadon, F., Lavrijsen, J., Leon-Carrion, J., Sannita, W. G., et al. (2010). Unresponsive wakefulness syndrome: a new name for the vegetative state or apallic syndrome. BMC Med. 8:68. doi: 10.1186/1741-70158-68
Luo, H., and Poeppel, D. (2007). Phase patterns of neuronal responses reliably discriminate speech in human auditory cortex. Neuron 54, 1001-1010. doi: 10.1016/j.neuron.2007.06.004

Luo, H., Wang, Y., Poeppel, D., and Simon, J. Z. (2006). Concurrent encoding of frequency and amplitude modulation in human auditory cortex: MEG evidence. J. Neurophysiol. 96, 2712-2723. doi: 10.1152/jn.01256.2005

Marino, S., Bonanno, L., and Giorgio, A. (2016). Functional connectivity in disorders of consciousness: methodological aspects and clinical relevance. Brain Imaging Behav. 10, 604-608. doi: 10.1007/s11682-015-9417-1

Maxwell, W. L., MacKinnon, M. A., Smith, D. H., McIntosh, T. K., and Graham, D. I. (2006). Thalamic nuclei after human blunt head injury. J. Neuropathol. Exp. Neurol. 65, 478-488. doi: 10.1097/01.jnen.0000229241.28619.75

Millman, R. E., Prendergast, G., Kitterick, P. T., Woods, W. P., and Green, G. G. (2010). Spatiotemporal reconstruction of the auditory steady-state response to frequency modulation using magnetoencephalography. Neuroimage 49, 745758. doi: 10.1016/j.neuroimage.2009.08.029

Monti, M. M., Rosenberg, M., Finoia, P., Kamau, E., Pickard, J. D., and Owen, A. M. (2015). Thalamo-frontal connectivity mediates top-down cognitive functions in disorders of consciousness. Neurology 84, 167-173. doi: 10.1212/wnl. 000000000001123

Monti, M. M., Vanhaudenhuyse, A., Coleman, M. R., Boly, M., Pickard, J. D., Tshibanda, L., et al. (2010). Willful modulation of brain activity in disorders of consciousness. N. Engl. J. Med. 362, 579-589. doi: 10.1056/nejmoa0905370

Naccache, L. (2018). Minimally conscious state or cortically mediated state? Brain 141, 949-960. doi: 10.1093/brain/awx324

Pan, J., Xie, Q., Qin, P., Chen, Y., He, Y., Huang, H., et al. (2020). Prognosis for patients with cognitive motor dissociation identified by brain-computer interface. Brain 143, 1177-1189. doi: 10.1093/brain/awaa026

Peelle, J. E., Gross, J., and Davis, M. H. (2013). Phase-locked responses to speech in human auditory cortex are enhanced during comprehension. Cereb. Cortex 23, 1378-1387. doi: 10.1093/cercor/bhs118

Pincherle, A., Johr, J., Chatelle, C., Pignat, J. M., Du Pasquier, R., Ryvlin, P., et al. (2019). Motor behavior unmasks residual cognition in disorders of consciousness. Ann. Neurol. 85, 443-447. doi: 10.1002/ana.25417

Ross, B., Borgmann, C., Draganova, R., Roberts, L. E., and Pantev, C. (2000). A high-precision magnetoencephalographic study of human auditory steady-state responses to amplitude-modulated tones. J. Acoust. Soc. Am. 108, 679-691. doi: 10.1121/1.429600

Ross, B., Herdman, A. T., and Pantev, C. (2005). Right hemispheric laterality of human $40 \mathrm{~Hz}$ auditory steady-state responses. Cereb. Cortex 15, 2029-2039. doi: $10.1093 /$ cercor/bhi078

Schiff, N. D. (2015). Cognitive motor dissociation following severe brain injuries. JAMA Neurol. 72, 1413-1415. doi: 10.1001/jamaneurol.2015.2899

Schnakers, C., Vanhaudenhuyse, A., Giacino, J., Ventura, M., Boly, M., Majerus, S., et al. (2009). Diagnostic accuracy of the vegetative and minimally conscious state: clinical consensus versus standardized neurobehavioral assessment. BMC Neurol. 9:35. doi: 10.1186/1471-2377-9-35

Simon, J. Z., and Wang, Y. (2005). Fully complex magnetoencephalography. J. Neurosci. Methods 149, 64-73. doi: 10.1016/j.jneumeth.2005.05.005

Soddu, A., Vanhaudenhuyse, A., Bahri, M. A., Bruno, M. A., Boly, M., Demertzi, A., et al. (2012). Identifying the default-mode component in spatial IC analyses of patients with disorders of consciousness. Hum. Brain Mapp. 33, 778-796. doi: 10.1002/hbm.21249

Steinmann, I., and Gutschalk, A. (2011). Potential fMRI correlates of $40-\mathrm{Hz}$ phase locking in primary auditory cortex, thalamus and midbrain. Neuroimage 54, 495-504. doi: 10.1016/j.neuroimage.2010.07.064

Stender, J., Gosseries, O., Bruno, M. A., Charland-Verville, V., Vanhaudenhuyse, A., Demertzi, A., et al. (2014). Diagnostic precision of PET imaging and functional MRI in disorders of consciousness: a clinical validation study. Lancet 384, 514-522. doi: 10.1016/s0140-6736(14)60042-8

Stender, J., Mortensen, K. N., Thibaut, A., Darkner, S., Laureys, S., Gjedde, A., et al. (2016). The minimal energetic requirement of sustained awareness after brain injury. Curr. Biol. 26, 1494-1499. doi: 10.1016/j.cub.2016.04.024

van Erp, W. S., Lavrijsen, J. C., Vos, P. E., Bor, H., Laureys, S., and Koopmans, R. T. (2015). The vegetative state: prevalence, misdiagnosis, and treatment limitations. J. Am. Med. Dir. Assoc. 16, 85 e9-85 e14.

Wang, F., Di, H., Hu, X., Jing, S., Thibaut, A., Di Perri, C., et al. (2015). Cerebral response to subject's own name showed high prognostic value in traumatic vegetative state. $B M C$ Med 13:83. 
Wang, Y., Ding, N., Ahmar, N., Xiang, J., Poeppel, D., and Simon, J. Z. (2012). Sensitivity to temporal modulation rate and spectral bandwidth in the human auditory system: MEG evidence. J Neurophysiol 107, 2033-2041. doi: 10.1152/ jn.00310.2011

Wannez, S., Heine, L., Thonnard, M., Gosseries, O., Laureys, S., and Coma Science Group Collaborators. (2017). The repetition of behavioral assessments in diagnosis of disorders of consciousness. Ann Neurol 81, 883-889. doi: 10. 1002/ana.24962

Way, T. W., Sahiner, B., Hadjiiski, L. M., and Chan, H. P. (2010). Effect of finite sample size on feature selection and classification: a simulation study. Med Phys 37, 907-920. doi: 10.1118/1.3284974

Wu, M., Li, F., Wu, Y., Zhang, T., Gao, J., Xu, P., et al. (2020). Impaired Frontoparietal Connectivity in Traumatic Individuals with Disorders of Consciousness: A Dynamic Brain Network Analysis. Aging Dis 11, 301-314. doi: 10.14336/ad.2019.0606

Zhang, J. H., Han, X., Zhao, H. W., Zhao, D., Wang, N., Zhao, T., et al. (2018). Personalized prediction model for seizure-free epilepsy with levetiracetam therapy: a retrospective data analysis using support vector machine. Br J Clin Pharmacol 84, 2615-2624. doi: 10.1111/bcp.13720

Zion Golumbic, E. M., Ding, N., Bickel, S., Lakatos, P., Schevon, C. A., McKhann, G. M., et al. (2013). Mechanisms underlying selective neuronal tracking of attended speech at a "cocktail party". Neuron 77, 980-991. doi: 10.1016/j. neuron.2012.12.037

Conflict of Interest: The authors declare that the research was conducted in the absence of any commercial or financial relationships that could be construed as a potential conflict of interest.

Copyright (c) $2021 \mathrm{Xu}$, Zou, He, Wen, Li, Gao, Ding and Luo. This is an open-access article distributed under the terms of the Creative Commons Attribution License (CC BY). The use, distribution or reproduction in other forums is permitted, provided the original author(s) and the copyright owner(s) are credited and that the original publication in this journal is cited, in accordance with accepted academic practice. No use, distribution or reproduction is permitted which does not comply with these terms. 